\title{
BTLBO Based SPMUs Placement Optimization for Fault Observation in Power Grid
}

\author{
Abdelkader ZITOUNI and Hamid BENTARZI \\ Laboratory Signals and Systems, \\ IGEE, University M'hamed Bougara Boumerdes, 35000 \\ Boumerdes, Algeria, \\ Iss@univ-boumerdes.dz
}

\begin{abstract}
The placement of synchro-phasor measurement units in electric transmission systems has also gained much attention for enhancing the control as well as the protection scheme. In this research work, a binary teaching learning based optimization (BTLBO) algorithm for the optimal placement of synchro-phasor measurement units (SPMUs) is proposed. The optimal PMU placement problem is formulated to minimize the number of SPMUs installation subject to full network observability of the power system buses under fault conditions. The effectiveness of the proposed method is verified by the simulation of IEEE 14-bus benchmark system.
\end{abstract}

Keywords: Binary teaching learning technique, optimization, PMUs placement, fault, observability.

\section{INTRODUCTION}

In order to make the electrical power systems more reliable, stable, and controllable; state estimation of the transmission network is necessary [1]. The Synchro Phasor Measurement Unit (SPMU) is a tool for measuring the voltage and current those are synchronized by using the global positioning system (GPS) satellite [2]. The power station including the Phasor Data Concentrator (PDC), which is used for analyzing the PMU data, can receive the synchronous data from each SPMU in real time [3].

State estimation of power network may allow the scheduling generation and interchange; monitoring outages and scheduling alternatives; supervising scheduled outages; scheduling frequency and time corrections; coordinating bias settings; and emergency restoration of system [4]. This can be achieved either by state estimation algorithms [5] or by means of SPMUs with extreme precision, time synchronization, and excellent performance. Measuring state estimation is achieved through complex bus voltages [6] that enable the estimation of the neighborhood bus voltage magnitudes and angles by using the line flow measurement (both real and reactive power). The static and dynamic behaviors of the power grid may be obtained from the information gathered by

Cite this article as:

A. ZITOUNIU and H. BENTARZI, "BTLBO Based SPMUs Placement Optimization for Fault Observation in Power Grid ", Algerian Journal of Signals and Systems, Vol. 4, Issue 1, June 2019.pp: 18-24.
SPMUs.

Faults that occur in the transmission line may be either permanent or temporary $[7,8]$. Permanent fault may lead to a broken transmission line or a power generator malfunction; this causes different signals during the pre-fault and post-fault. This can be easily detected and located. However, the temporary fault may cause insulator flashover, which may lead to full insulator breakdown if it occurs frequently. This emphasizes the importance of SPMU-based fault location technique [9-11].

Nowadays, Wide Area Monitoring System (WAMS) that is considered the most advanced system to detect disturbances and avoid a bulk blackout is based on the SPMUs. It aims to maintain the dynamic stability in the whole power network [11]. This is implemented by synchronizing and recording the acquired data from systems in distributed locations through new computing and communication technologies. Upon their delivery to the central control station, these data are measured and analyzed from any point of the power network. In addition to its ability to monitor the static stability of the network (as traditional SCADA), WAMS enables the controllers to recognize unusual activities within the power network such as instability in the network voltage, to analyze the network oscillation, and to detect fault localization. Thus, using the data provided by SPMUs installed in some appropriate buses of a power grid, one can construct a new type of measuring system to improve the observability and the precision of the power system state estimator. The observability 
depends on the type, the number and the geographic distribution of measurements [12]. Several methods [13-17] were considered when formulating the optimal placement of monitoring devices for fault location.

In this research work, a binary teaching learning based optimization (BTLBO) algorithm for the optimal placement of SPMUs is proposed. The optimal SPMU placement problem is formulated to minimize the number of SPMUs installation subject to full network observability at the power system buses during the fault. The effectiveness of the proposed method is verified by the simulation of IEEE14-bus.

\section{FAULT ANALYSIS USING SPMU}

SPMU-based fault location technique is achieved through monitoring the synchronized fault voltages, calculating the line currents between these buses, and forming bus injection currents at two terminals of the faulted line. Thus, calculation of fault locations can be indirectly investigated.

A SPMU placed at a given bus of the network can measure both the phasor voltages and phasor currents of all lines incident to that bus. Thus, the entire parameters of a bus can be made observable by placing judiciously SPMUs at specific buses of the network. As shown in Fig.1, most power transmission networks may consist in general of five different sub-network configurations or types [18]. In this figure, for each of the five subnetworks, the placed SPMUs obey to what is called the "one bus spaced deployment strategy".

Whatever is the considered sub-network type, it is always possible to make the fault location problem of that sub-network similar to the fault location problem of type- 1 subnetwork [19]. A faulty type-1 sub-network is shown on Fig.2. In this figure, a fault occurs in line jk between bus $\mathrm{j}$ and bus $\mathrm{k}$. Thus, the fault location problem consists of finding the parameter $D_{k}$.

By using the voltage phasor of bus $i$ and the parameters of line $\mathrm{ij}$, the voltage phasor of bus $\mathrm{j}$ will be determined as:

$\left.=V_{i}-\left(I_{i j}-V_{i}-L_{i j} Y_{i j} / 2\right) L_{i j} Z_{i j} \quad\right)$

In addition,

$=I_{k f}+I_{j f}$

Where the line parameters $Z_{i j}$ and $Y_{i j}$ are relative to the $\Pi$ line model.

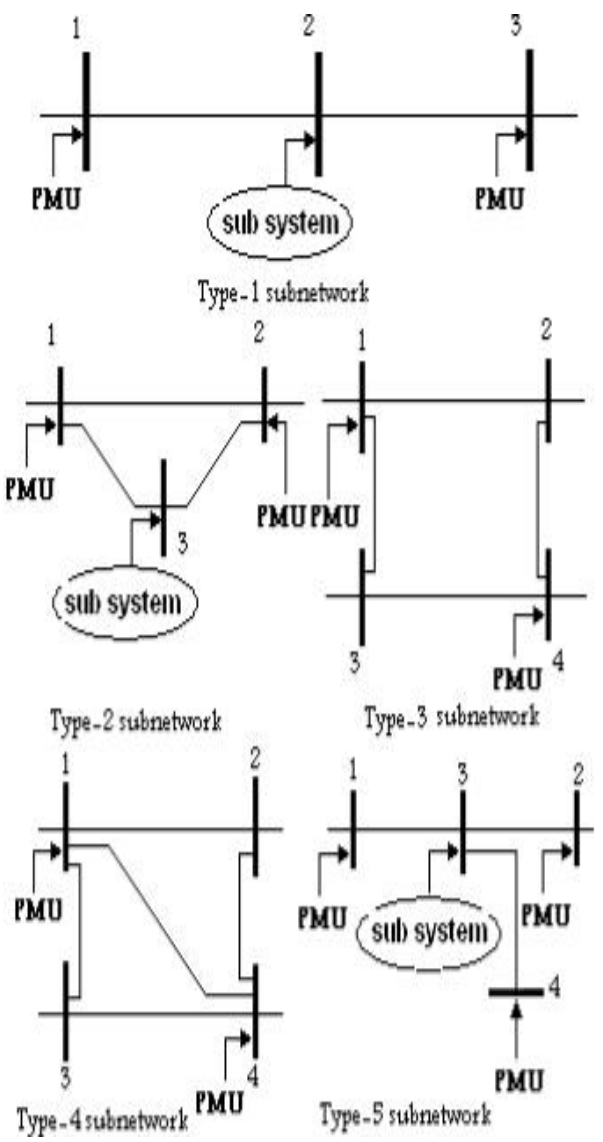

Fig. 1 The five possible sub-network configurations.

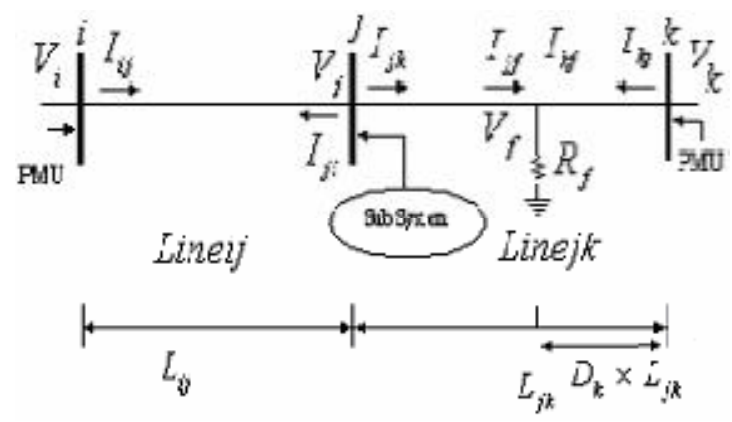

Fig. 2 Faulty type-1 sub-network

Current phasor ljk however, cannot be deduced with the similar way as no SPMU is connected at bus $\mathrm{j}$. Therefore, the problem consists of finding two unknown variables $\mathrm{I}_{\mathrm{jk}}$ and $D_{k}$. Using the basic electrical networks theory and assumption constraints, the two sets of equations of the two unknown variables can be derived:

Network equations, derived from equations (1) and (2)

$F_{1}\left(l_{j k}, D_{k}, \theta\right)=0$ 
SUMMARY OF THE TWO GROUPS OF EQUATIONS FOR FAULT LOCATION DETECTION

\begin{tabular}{|c|c|}
\hline \multicolumn{2}{|c|}{ Group 1: Network equation $(n=0,1,2)}$, \\
\hline \multicolumn{2}{|c|}{$\begin{array}{c}\mathrm{Vfn}=\mathrm{Vkn}-(\mathrm{Ikjn}-\mathrm{Vkn} \times \mathrm{Ykfn} / 2) \times \mathrm{Znfn} \\
\mathrm{Ijkn}=(\mathrm{Vjn}-\mathrm{Vfn}) / \mathrm{Zjfn}+\mathrm{Vjn} \times \mathrm{Yjfn} / 2 \\
\mathrm{Ifn}=(\mathrm{Vkn}-\mathrm{Vfn}) / \mathrm{Zkfn}-\mathrm{Vfn} \times \mathrm{Ykfn} / 2+ \\
(\mathrm{Vjn}-\mathrm{Vfn}) / \mathrm{Zjfn}-\mathrm{Vfn} \times \mathrm{Yjfn} / 2\end{array}$} \\
\hline \multicolumn{2}{|r|}{ Group 2: Constraint equations } \\
\hline $\begin{array}{c}\text { a-g } \\
\text { fault }\end{array}$ & $\begin{array}{c}\operatorname{Re}(\text { If0 }+ \text { If1 }+ \text { If } 2) \times \operatorname{Im}(V f 0+V f 1+ \\
\text { Vf2 })= \\
\operatorname{Im}(\text { If0 }+ \text { If1 + If2 }) \times \operatorname{Re}(V f 0+V f 1+ \\
\text { Vf2 })\end{array}$ \\
\hline $\begin{array}{l}\text { b-c-s } \\
\text { fault }\end{array}$ & $\begin{array}{c}\operatorname{Re}(\text { If1 }- \text { If2 }) \times \operatorname{Im}(V f 1-V f 2)= \\
\operatorname{Im}(\text { If1 }- \text { If2 }) \times \operatorname{Re}(V f 1-V f 2)\end{array}$ \\
\hline $\begin{array}{l}\text { b-c-g } \\
\text { fault }\end{array}$ & $\mathrm{Vf1}=\mathrm{Vf2}$ \\
\hline $\begin{array}{l}\text { a-b-c- } \\
\text { g } \\
\text { fault }\end{array}$ & $\operatorname{Re}($ If 1$) \times \operatorname{Im}($ Vf1 $)=\operatorname{Im}($ If1 $) \times \operatorname{Re}(\mathrm{Vf} 1)$ \\
\hline
\end{tabular}

Constraints equations, assuming pure resistance fault impedance or $\operatorname{Arg}($ lf $)=\operatorname{Arg}$ (Vf)

$F_{2}\left(l_{j k}, D_{k}, \theta\right)=0$

Where $\theta$ represents the known variables, such as $\mathrm{V}_{\mathrm{i}}, \mathrm{V}_{\mathrm{i}}, \mathrm{V}_{\mathrm{k}}$, etc.

In Table 1 are summarized the two groups of equations for fault location detection of three phase faults.

solving numerically these sets of equations leads to the unknown parameter Dk, that is the location of the fault on the line between bus $\mathrm{j}$ and bus $\mathrm{k}$.

\section{BROBLEM FORMULATION}

It is not economical to install a PMU at each bus of a wide-area network. Thus, the problem of optimal SPMU placement (OSP) concerns with where and how many SPMUs should be implemented in a power grid to achieve full observability at minimum number of SPMUs [4].

A SPMU placement optimization problem, for n-bus system, may be defined as:

Objective function: $J=\operatorname{Min} \sum_{i=1}^{n} x_{i}$

such that: $f(X) \geq \hat{i}$

(3)

Where $\mathrm{x}$ is a binary decision variable vector, whose entries are given as:

$$
x_{i}=\left\{\begin{array}{rr}
1 & \text { if Busihas a PMU } \\
0 & \text { otherwise }
\end{array}\right.
$$

$f(X)$ is a vector function, whose entries are non-zero if the corresponding bus voltage can be obtained by using the given measurement set, otherwise zero. Entries of a vector î are all equal to two or more.

\section{Constraints}

In order to form the constraint set, entries of the binary connectivity matrix $A$ are defined as follows:

$$
A_{k, m}=\left\{\begin{array}{llc}
1 & \text { if } & k=m \\
1 & \text { if } & k \text { and } m \text { are connected } \\
0 & \text { if } & \text { otherwise }
\end{array}\right.
$$

According to the knowledge that by placing a PMU on one bus, the voltage phasors of neighboring buses may be determined by:

$$
\mathrm{f}=\mathrm{A} \times \mathrm{X}
$$

Constraint functions defined by Equations (3), (4) and (5) may satisfy full network fault observability even with optimization of the number of PMUs.

The method for making the constraint equations (vector function $f(X)$ ) can be discussed for the following cases:

Neither PMU measurements nor conventional (flow and injection) measurements exist,

Only flow measurements exist,

Both flow and injection measurements (they may be zero or measured injections) exist or PMU and conventional flow or injection measurements exist.

To verify the proposed method, it has been tested using IEEE 14-bus grid shown in Fig.3.

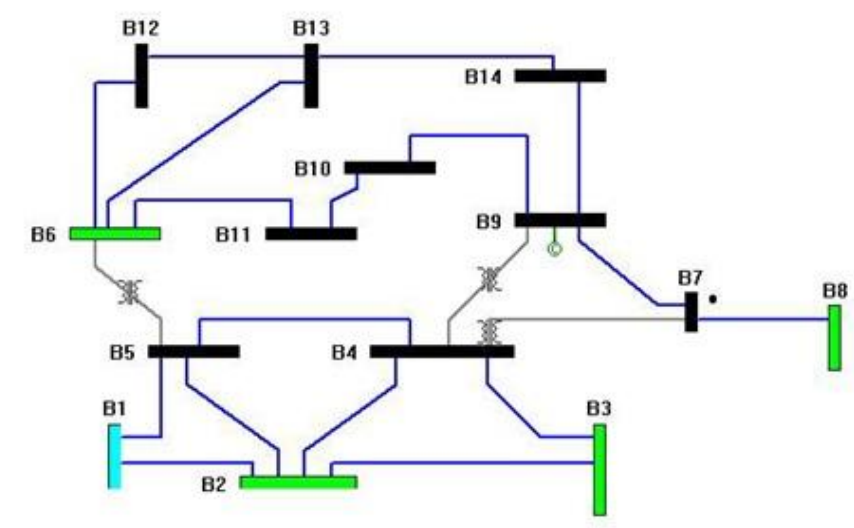

Fig. 3 IEEE 14-bus system

\section{BTLBO OPTIMIZATION ALGORITHM}

A new method that is binary version of TLBO has been used in this paper [20]. The 
algorithm of this binary version can be discussed as:

Step 0: define an optimization problem, the objective function:

Minimize (objective function) $y=$ $\mathrm{f}\left(\mathrm{x}_{1}, \mathrm{x}_{2}, \ldots, \mathrm{x}_{\mathrm{n}-1}, \mathrm{x}_{\mathrm{n}}\right)$ such that: $\mathrm{x}_{1}, \mathrm{x}_{2}, \ldots, \mathrm{x}_{\mathrm{n}-1}, \mathrm{x}_{\mathrm{n}}$ can take only two values: either a " 1 " or a " 0 ".

choose the number of students will be used or the population size, elite size and the number of generation. It can be considered that the minimization problem; the maximization is similar.

Step 1: initialization: propose a population (that will be made to attain the final solution) or students randomly according to the following equation:

$$
x_{(i, j)}^{1}=\operatorname{randi}([0,1], 1)
$$

i: refer to student number, so there are ith student, $i=1,2, \ldots, P$

$\mathrm{j}$ :refer to the course number, $x_{(\mathrm{i}, \mathrm{j})}$ is the level of the ith student at the jth course, $j=1,2, \ldots, D$ The small number 1 refers to the generation number that is the first generation.

After the manipulation of the above equation $\mathrm{P} \times \mathrm{D}$ times, we obtain a $\mathrm{P} \times \mathrm{D}$ matrix which represents our population:

$$
\text { population }^{1}=\left[\begin{array}{ccc}
x_{(1,1)}^{1} & \cdots & x_{(1, D)}^{1} \\
x_{(2,1)}^{1} & \ddots & x_{(2, D)}^{1} \\
\vdots & \ddots & \vdots \\
x_{(P, 1)}^{1} & \cdots \theta & x_{(P, D)}^{1}
\end{array}\right]
$$

(8)

Choose the teacher: the best student is the one which has the minimum fitness function.

Step 2: teacher phase

Every student tries to be as his teacher, so he makes his design variables as those of the teacher, of course not all of them otherwise he will be exactly as his teacher. Thus, the student copies some components of teacher and replaces his components by copied ones. The number of copied components depends on the ability of teacher and the student; that is represented by one random number $\mathrm{h}$. The number $\mathrm{h}$ that is between 0 and $D$ is given by:

$$
h=\operatorname{randi}(D, 1)
$$

This number determines how many courses that student ${ }^{1}$ will learn from his teacher. The location of these courses in the teacher vector can be specified according to the following equation:

$$
\text { Course }=\operatorname{randi}(h,[1, h])
$$

These randomly selected courses are now ready to be transmitted from teacher to student:

$$
\begin{aligned}
& X_{i, \text { new }}=X_{i} \\
& X_{i, \text { new }}(\text { course })=X_{\text {teacher }}(\text { course }) \\
& \text { if } f\left(X_{i, \text { new }}\right)<f\left(X_{i}\right) \text { then } X_{i}=X_{i, \text { new }} \text { else do nothing }
\end{aligned}
$$

Execution may make duplicate solutions; consequently one more step is required.

Step 3: remove duplicate solutions by mutation on randomly selected dimensions.

Step 4: learner phase

The interaction between students in binary interpretation will be as follows:

Select for a student $\mathrm{i}$ another one $\mathrm{j}$, if $\mathrm{j}$ is better than $i$ then $i$ will learn from $j$ by trying to change components that are different from those of $\mathrm{j}$, in order to be similar to him; otherwise he will change some components that are similar to those of $\mathrm{j}$. This can be done as follows:

For $\mathrm{i}=1: \mathrm{P}$ do

Select another student j and record where they are similar and where they are different in a vector,

$$
\begin{array}{r}
\mathrm{q}=\left[\begin{array}{ll}
\mathrm{q}_{1} \mathrm{q}_{2} \ldots \ldots \ldots \mathrm{q}_{\mathrm{D}}
\end{array}\right] \text { such that } \mathrm{q}_{\mathrm{k}}=1 \text { if } \mathrm{x}_{\mathrm{i}, \mathrm{k}}^{\mathrm{g}} \\
=\mathrm{x}_{\mathrm{j}, \mathrm{k}}^{\mathrm{g}} \text { else } \mathrm{q}_{\mathrm{k}}=0 ; \mathrm{k}=1, \ldots, \mathrm{D}
\end{array}
$$

The vector $h=\operatorname{randi}(D, 1)$ states how many courses student $i$ will learn from $j$ if he is better than him or change them in other case. The vector course $=\operatorname{randi}(\mathrm{h},[1, \mathrm{~h}])$ gives the location of courses which will be learnt or changed.

Now the learning process can be implemented:

$\mathrm{X}_{\mathrm{i} \text {,new }}=\mathrm{X}_{\mathrm{i}}$, the new student is the old one with some modifications.

if $f\left(X_{i}^{g}\right)<f\left(X_{j}^{g}\right)$ then $X_{i, n e w}($ course $)=X_{j}($ course $) \times n o t(q($ course $))$

else if $f\left(X_{i}^{g}\right) \geq f\left(X_{j}^{g}\right)$ then $X_{i, \text { new }}$ (course $)=\operatorname{not}\left(X_{j}(\right.$ course $\left.)\right) \times q($ course $)$ (12)

Equation (12) shows that some components where $\mathrm{i}$ and $\mathrm{j}$ differs will be copied from $\mathrm{j}$ (the highest learned one between the two) to i otherwise ( $j$ is inferior to $i$ ) some of similar component in i will be changed from 0 to 1 or 1 to 0 . Now, check if the new student is better than the old:

$$
\text { if } f\left(X_{i, \text { new }}\right)<f\left(X_{i}\right) \text { then } \mathrm{X}_{i}=X_{i, \text { new }} \text { else do nothing }{ }_{(13)}
$$

After the implementation of the process for all the population, a new teacher will be determined.

Step 5:-Remove duplicate solutions.

-replace bad solutions by elite solutions and again remove duplicate solutions.

Step 6: if $g \neq$ number of generation then go to step2 else stop. 
Using the binary teaching learning based optimization explained previously which is based on the problem formulation pointed out in previous section; an algorithm for the optimal placement problem is given as follows:

1. Construct the binary connectivity matrix using the one line diagram and constraint modification when injection, flow measurements or already installed PMUs.

2. Define the optimization parameters; the population size (pop_size), the design variables ( $N$ buses), and the number of generation (N_gen), the (elite size).

3. Generate random solutions within boundaries of the system.

4. Check that random solutions satisfy the inequality constraint of buses $f(x)=\left(A^{*} X\right) \geq \hat{\imath}$,

5. Modify those are not satisfying the constraints.

6. Calculate the fitness of each solution using the objective function of expression,

7. Set the best solution as teacher of the population,

8. For each student, apply teacher phase, replace $x$ by $x_{n e w}$ if it gives better fitness function (less) otherwise keep the old one.

9. If the duplicate solutions exist then modify the duplicate solutions for avoiding the trapping in the local optima. In the present work, duplicate solutions are modified by mutation on randomly selected dimensions of the duplicate solutions before executing the next generation without losing the observability.

10.For each student apply learner phase, replace $x$ by $x_{n e w}$ if it gives better fitness function (less) otherwise keep the old one.

11. Again, remove duplicate solutions keeping the constrained satisfied (observability).

12.Replace (elite_size) bad solutions by (elite_size) elite solutions.

13.Again, remove duplicate solutions keeping the constrains satisfied (observability), then determine the teacher.

14. Repeat from step 7 for maximum number of iterations.

15.Put the best solution $x_{\text {teacher }}$ as the final solutions.

The flowchart for the optimal placement algorithm is shown in Figs. 4 and 5.

\section{RESULTS AND DISCUSSION}

The proposed SPMU placement optimization algorithm has been implemented using MATLABß on the IEEE 14-bus network shown in Fig.3. Network data is given in Table I. The used parameters in the optimization are: The maximum iteration number in BTLBO is set at 1000 with the population size of 100 . In this simulation, the best solutions of the binary TLBO is obtained after 20 runs of the algorithm. The simulations have been carried out on a PC Intel i5 $(2.40 \mathrm{GHz})$ with $4 \mathrm{~GB}$ RAM. It may be mentioned that the required number of SPMUs is not necessarily unique. Since heuristic algorithms, such as TLBO, are based on a random search in the search space of the problem, and the result of each execution of these algorithms might be different from another one. Therefore, they must be run several times to ensure that the optimal point of the problem can be attained. However, from the observability point of view, there is no difference between different configurations with the same number of SPMUs, so only one configuration has been presented.

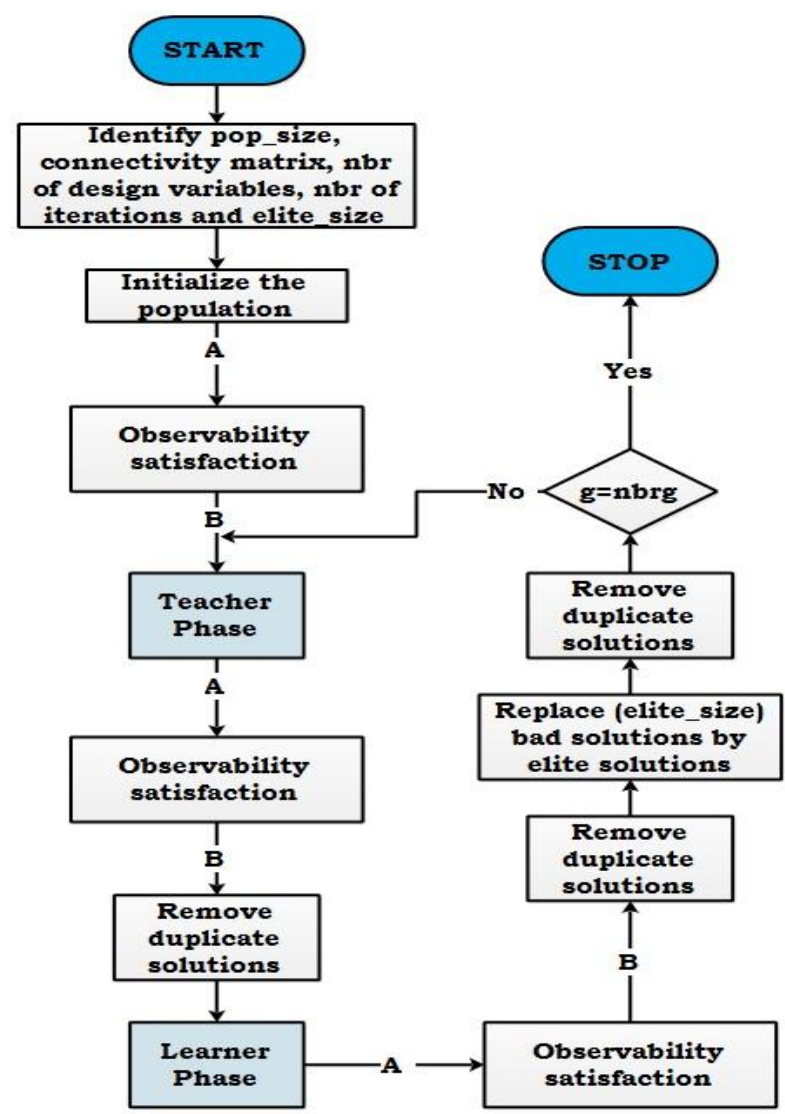

Fig. 4: Flow chart of BTLBO algorithm applied to the optimal SPMU placement 


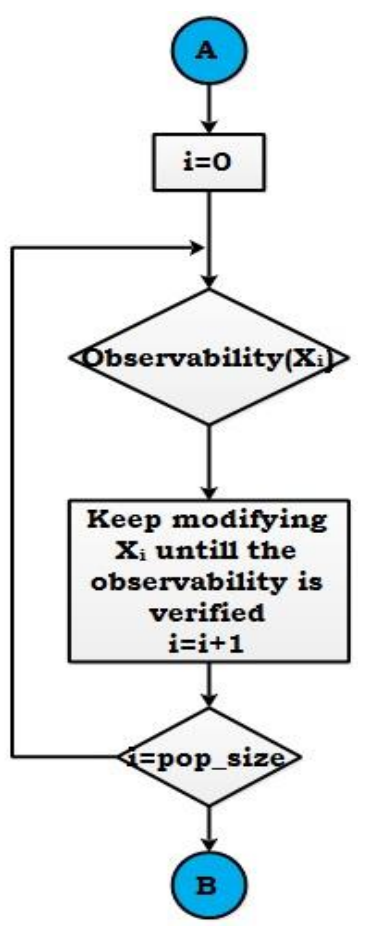

Fig. 5: Observability subroutine

SYSTEM INFORMATION OF IEEE 14-BUS SYSTEM.

\begin{tabular}{|c|c|c|c|}
\hline System & $\begin{array}{c}\text { Num. of } \\
\text { branches }\end{array}$ & $\begin{array}{c}\text { Num. of } \\
\text { zero } \\
\text { injections }\end{array}$ & $\begin{array}{c}\text { Zero } \\
\text { injection } \\
\text { buses }\end{array}$ \\
\hline $\begin{array}{c}\text { IEEE } \\
\text { 14-bus }\end{array}$ & 20 & 1 & 7 \\
\hline
\end{tabular}

OPTIMUM NUMBER AND LOCATION OF SPMU

\begin{tabular}{|c|c|c|}
\hline System & $\begin{array}{c}\text { Num. of } \\
\text { PMUs }\end{array}$ & $\begin{array}{c}\text { Location of } \\
\text { PMUs }\end{array}$ \\
\hline $\begin{array}{c}\text { IEEE 14-bus with } \\
\begin{array}{c}\text { Zero injection } \\
\text { buses }\end{array}\end{array}$ & 7 & $1,2,4,6,9,10,13$ \\
\hline $\begin{array}{c}\text { IEEE 14-bus with } \\
\text { injection buses }\end{array}$ & 8 & $\begin{array}{c}1,2,4,6,9,10,13, \\
14\end{array}$ \\
\hline
\end{tabular}

Two sets of simulations have re carried out on the IEEE 14- bus system, which are initially assumed to have no flow measurements and no previously existed SPMUs. In the first set of simulations, zero injection is simply ignored while in the second set, it is used as existing measurement.

The IEEE 14 bus test system has only one zero-injection bus. The output of the BTLBO algorithm is 8 SPMUs. The information of the system and zero injections are given in Table II.

The global solution may be obtained after a few iterations (between 5 and 10) and population size (10). Seven SPMUs can be installed in order to fully observe the IEEE14 bus system. However, when injection is ignored, different configuration of PMUs installation is obtained, whereas, considering injection gives only one set $(1,2,4,6,9,10$ and 13). It can be noted that the number of SPMUs required for system fault observability reduces by 1 , if the system observability is to be maintained after considering zero injection buses.

The number of SPMUs calculated by the proposed approach is given in Table III. The number of SPMUs that guarantees full observability of power system during any failure that can occur; each bus is observable by at least two SPMUs.

\section{CONCLUSIONS}

The obtained simulation results are in agreement with the previous research work results $[13,14,16$, and 17]. Moreover, the results show that full network observability can be achieved under fault conditions using more than 50\% PMUs installation; however, under normal conditions, approximately $30 \%$ SPMUs installation is required.

\section{References}

K.J. Karimi, Power system static state estimation with phasor measurements. Cornell Univ., Ithaca, NY (USA), 1986.

A.G. Phadke, Synchronized phasor Computer Applications in power, 6, pp 10-15, 1993.

A. Ouadi, H. Bentarzi and J. C. Maun, Phasor Measurement Unit Reliability Enhancement Using Real-Time Digital Filter, International Journal of Circuits, Systems and Signal processing, 5, pp 18, 2011.

A.G.Phadke, and J.S. Thorp, Synchronized phasor measurements and their applications. Vol. 1.: Springer, 2008.

J. Allemong, L. Radu, and A. Sasson, A fast and reliable state estimation algorithm for AEP's new control center. IEEE Transactions on Power Apparatus and systems, 4, pp 933-944, 1982.

N.H. Abbasy, and H.M. Ismail, A unified approach for the optimal PMU location for power system state estimation. IEEE Transactions on Power Systems,. 24, pp 806813, 2009.

H. Yin, and L. Fan. PMU data-based fault location techniques. in North American Power Symposium (NAPS), . IEEE, 2010.

Y.-H. Lin, C.-W. Liu, and C.-S. Chen, A new PMU-based fault detection/location technique for transmission lines with consideration of arcing fault discrimination-part I: theory and algorithms. IEEE Trans. on power delivery, 19, pp 1587-1593, 2004.

Y.-H. Lin, C.-W. Liu, and C.-S. Chen, A new PMU-based fault detection/location 
technique for transmission lines with consideration of arcing fault discrimination-partll: performance evaluation. IEEE Trans. on power delivery. 19, pp 1594-1601, 2004.

A. Ibe, and B. Cory, A travelling wave-based fault locator for two-and three-terminal networks. IEEE Trans. on power delivery, 1, 283-288, 1986.

T. Takagi, et al., Development of a new type fault locator using the one-terminal voltage and current data. IEEE Transactions on Power apparatus and systems, 8, 2892-2898, 1982.

Xu Bei, Yeo Jun Yoon and Ali Abur, Optimal placement and utilization of phasor measurements for state estimation, Texas A\&M University, 2008.

S. Chakrabarti, E. Kyriakides, Optimal placement of phasor measurement units for power system observability, IEEE Trans. Power Syst. 23, 14331440, 2008.

H.H. Müller, C.A. Castro, Genetic algorithm-based phasor measurement unit placement method considering observability and security criteria, IET Gener.Transm. Distrib. 10, 1, 270-280, 2016.

N.H. Abbasy, H.M. Ismail, A unified approach for the optimal PMU location for power system state estimation, IEEE Trans. Power Syst. 24, 806813, 2009.

M. Hajian, et al., Optimal placement of PMUs to maintain network observability using a modified BPSO algorithm, Int. J. Electr. Power 33, 28-34, 2011.

A. Mahari, S. Heresh, Optimal PMU placement for power system observability using BICA considering measurement redundancy, Electr. Power Syst. Res. 103, 78-85, 2013.

Kai-Ping Lien, Chih-Wen Liu, Chi-Shan Yu, and Joe-Air Jiang, "Transmission Network Fault Location Observability With Minimal PMU Placement," IEEE Transaction on Power Delivery, Volume 21, Issue 3, July 2006 Page(s):1128 - 1136.

S. S. Geramian, H. Askarian Abyane, Member IEEE, K. Mazlumi," Determination of Optimal PMU Placement for Fault Location Using Genetic Algorithm", 13th International Conference on Harmonics and Quality of Power, ICHQP 2008, Wollongong, NSW, Australia.

A. Recioui, H.Bentarzi and A. Ouadi, Application of a Binary Teaching Learning-Based Algorithm to the Optimal Placement of Phasor Measurement Units, Book chapter, Progress in Clean Energy, 1, 817-830, 2015. 